\title{
Effects of dental treatment and systemic disease on oral health-related quality of life in Korean pediatric patients
}

\author{
Ji-Soo Song ${ }^{1}$, Hong-Keun Hyun ${ }^{2}$, Teo Jeon Shin ${ }^{2}$ and Young-Jae Kim² ${ }^{2 *}$
}

\begin{abstract}
Background: The findings that not only dental caries but also systemic disease can exert a negative effect on oral health-related quality of life (OHRQoL), and that dental treatment can improve OHRQoL have been confirmed in multiple studies. The purpose of this study is to investigate the impact of dental treatment on OHRQoL of Korean pediatric patients and the differences in OHRQoL between patients with and without systemic disease.

Methods: All the primary caregivers of pediatric patients who underwent dental treatments under either general anesthesia or intravenous deep sedation at Seoul National University Dental Hospital completed abbreviated versions of the Child Oral Health Impact Profile (COHIP-14) and Family Impact Scale (FIS-12) surveys on OHRQOL pre- and posttreatment (average: $2.4 \pm 1.7$ months after dental treatment). This is a case control study with patients divided into two groups according to the presence or absence of systemic disease.

Results: Data from 93 pediatric patients (46 male and 47 female, average patient age: $5.0 \pm 3.4$ years) were analyzed to compare OHRQoL before and after treatment with the Wilcoxon signed-rank test and to calculate the effect size using Cohen's d. All of the patients exhibited an improvement in OHRQoL (COHIP-14: $p<0.001$, effect size $=1.0$; FIS-12: $p<0.001$, effect size $=0.7$ ). Patients with systemic diseases demonstrated lower OHRQoL in both pre- and posttreatment surveys than patients without systemic diseases (Wilcoxon Rank-sum test, both COHIP-14 and FIS-12: $p<0.05)$. The COHIP-14 appears to have a greater impact on the FIS-12 in patients with systemic disease than those without (explanatory power of 65.3 and $44.6 \%$, respectively).
\end{abstract}

Conclusions: Based on the primary caregivers' perceptions, dental treatment can improve the OHRQoL in Korean pediatric patients. Systemic disease results in a reduced OHRQoL, and the awareness of patients' oral health appeared to have a greater impact on OHRQoL for family members of patients with a systemic disease.

Trial registration: KCT0002473 (Clinical Research Information Service, Republic of Korea) and 22 Sep 2017, retrospectively registered.

Keywords: Child, Dental treatment, Systemic disease, Oral health-related quality of life

\footnotetext{
* Correspondence: neokarma@snu.ac.kr

${ }^{2}$ Department of Pediatric Dentistry, Dental Research Institute, School of

Dentistry, Seoul National University, Seoul National University Dental Hospital,

101, Daehakno, Jongno-gu, 03080 Seoul, Republic of Korea

Full list of author information is available at the end of the article
}

(c) The Author(s). 2018 Open Access This article is distributed under the terms of the Creative Commons Attribution 4.0 International License (http://creativecommons.org/licenses/by/4.0/), which permits unrestricted use, distribution, and reproduction in any medium, provided you give appropriate credit to the original author(s) and the source, provide a link to the Creative Commons license, and indicate if changes were made. The Creative Commons Public Domain Dedication waiver (http://creativecommons.org/publicdomain/zero/1.0/) applies to the data made available in this article, unless otherwise stated. 


\section{Background}

Dental caries is the most common chronic oral disease, with a high prevalence in children and adolescents worldwide [1]. The prevalence of active caries in primary teeth was as high as $34.5 \%$, and that of dental caries experience in primary teeth was as high as $62.2 \%$ in 2012 Korean study [2]. Oral health-related quality of life (OHRQoL) is a multidimensional concept that includes a subjective evaluation of the individual's oral health status, functional well-being, social and emotional well-being, expectations of and satisfaction with care, and sense of self-image [3]. Its importance is widely emphasized in both research and clinical settings, given the increasing demand for active participation of patients in the treatment process, and the lack of basic treatment for certain chronic diseases (e.g., dental caries, periodontal disease) that require long-term treatment and follow-up. Nevertheless, research about the OHRQoL of pediatric patients in Korea has only recently been initiated despite the high prevalence of dental caries. The only study that has conducted a full-scale reliability and validity test in Korea was reported by Ahn et al., in which a Korean version of the Child Oral Health Impact Profile (COHIP) was used in 2236 children and adolescents aged $8-15$ years [4].

The findings that dental caries can exert a negative effect on OHRQoL and that dental treatment can improve OHRQoL have been confirmed in several studies [5-9]. Pain caused by dental caries can interfere with normal masticatory function and sleep, which can inhibit normal body growth [10]. Unpleasant smiles associated with the destruction of tooth structure also can negatively influence the social life of children [11]. In addition, the perceptions and attitudes of primary caregivers on oral health influence the behavioral patterns regarding their child's oral health [12]. Chronic disease such as dental caries in children can affect family life $[13,14]$, and patients with systemic disease have been shown to have low OHRQoL [15-17]. Their underlying disease may be associated with poor oral health, but they may also have difficulties maintaining their oral health and accessing adequate dental care due to underlying disease [18]. There are no studies that have been conducted to identify the relationship between dental caries and the OHRQoL of pediatric patients and their families, and to compare the OHRQoL between patients with and without systemic diseases in Korea. Therefore, this study examined the impact of dental treatment on OHRQoL of Korean pediatric patients and the differences in the OHRQoL between patient with and without systemic disease.

\section{Methods}

\section{Subjects}

This study involved all primary caregivers or parents of pediatric dental patients who underwent dental treatment under either general anesthesia or intravenous deep sedation at Seoul National University Dental Hospital pediatric department from February 2013 to February 2014. Five professors who were all experienced in general anesthesia and intravenous sedation in the pediatric dentistry department conducted all the dental treatments, and standardized treatment protocols were followed. Patients who received dental treatment without general anesthesia or intravenous sedation were not included in the study. The use of general anesthesia or intravenous sedation is decided by the anesthesiologist based on the physical condition of the airway and respiratory system, not on the severity of dental caries. Primary caregivers accompanying the patients on the day of treatment were invited to participate in the survey. The study was performed with the approval of the Seoul National University Dental Hospital Research Ethics Board (IRB Number: CRI12006). We fully explained the study to the primary caregivers only if they were the legal guardians of the patients and only included participants with written consent on the day of treatment.

\section{Study design}

This study was a case control study to compare OHRQoL between the patients with and without systemic disease in each cohort. Accordingly, patients were categorized into two groups. The patient group without systemic disease did not exhibit conditions that encumbered everyday life, but required either general anesthesia or intravenous deep sedation due to dental phobia and a large number of dental caries. The group with systemic disease included patients with special health care needs, such as intellectual disability (ID), autism, or developmental disorders, as well as conditions that affect everyday life (e.g., cancer, cerebral palsy, convulsive disorders, genetic disorders, and cardiovascular disorders) $[19,20]$. Cases with dental treatments that did not involve pulp treatment or restorations-including periodontal treatment, such as scaling, or minor oral surgery, such as removal of supernumerary teeth-were excluded from the study.

Primary caregivers were asked to fill out surveys on OHRQoL, pre-treatment as well as post-treatment when the patients returned for a follow-up visit. As calculated from our pilot study performed in the initial stage of this study with 20 patients (10 patients without systemic disease and 10 patients with systemic disease), the power calculation indicated that 104 cases were required to compensate for a $20 \%$ drop-out rate at $5 \%$ significance level and $80 \%$ statistical power. The pre-treatment survey included responses from 109 cases and follow-up post-treatment surveys were completed for 93 of these cases within 6 months. These cases were selected for 
analysis. Sixteen cases were excluded, as the patient did not have a follow-up appointment, a different primary caregiver accompanied the patient for the post-treatment visit, or the primary caregiver declined to complete the post-treatment survey. The post-treatment survey was completed in an average of $2.4 \pm 1.7$ months after dental treatment.

\section{Surveys}

In order to assess OHRQoL, the Child Oral Health Impact Profile (COHIP) and Family Impact Scale (FIS) were utilized. An abbreviated version of the COHIP, "COHIP-14", which included 10 items from the Oral Health subscale $(\mathrm{OH})$ and 4 items from the Functional Limitation subscale (FL), was used in this study [21]. Similarly, the "FIS-12" scale used in the study included 5 items from the Parental/Family Activity subscale (PA), 4 items from the Parental Emotion subscale (PE), 2 items from the Family Conflict subscale (FC), and 1 item from the Financial Burden subscale (FB) [21]. Because pediatric patients requiring general anesthesia or intravenous sedation were usually younger than the target age of COHIP and FIS, these subscales and items were selectively chosen from the original questionnaires to be reasonably assessed among the primary caregivers of these patients. Several items that caregivers could not answer correctly or required an active response from the patient were removed through an active discussion between two experienced dentists [21]. The resultant subscales and items are outlined in Tables 1 and 2 . The pre-treatment COHIP-14 survey assessed the frequency of issues arising from dental disorders in pediatric patients from the primary caregiver's perspective, and the FIS-12 assessed the impact on everyday life activities and emotions of the patient and family members in the 3 months prior to the survey. For the post-treatment survey, the primary caregivers were instructed to reflect on changes post-treatment when completing both the COHIP-14 and FIS-12. Both measurements utilized a 5-point Likert scale, where the COHIP-14 and the FIS-12 ranged from 0, being "Never", to 4, being "Almost every day". Because the items of COHIP-14 were negatively worded, the scores in COHIP-14 were reversed [22]. The scores of the items were added to calculate subscale scores, which were then summed to obtain the finalized COHIP-14 and FIS-12 scores. The COHIP-14 score ranged from 0 to 56, while FIS-12 score ranged from 0 to 48 . Higher COHIP scores and lower FIS scores corresponded to a better OHRQoL.

In addition to the COHIP and FIS, global ratings of OHRQoL also known as single-item ratings, were used to assess the general oral health of the pediatric patients and their overall QoL. These questions were answered

Table 1 Prevalence and mean values of the 14-item Child Oral Health Impact Profile (COHIP-14) scores before and after dental treatment (average $2.4 \pm 1.7$ months' follow-up period) $(n=93)$

\begin{tabular}{|c|c|c|c|c|c|}
\hline & \multirow{2}{*}{$\begin{array}{l}\text { Before COHIP-14 score } \\
\text { Mean(SD) }\end{array}$} & \multirow{2}{*}{$\begin{array}{l}\text { After COHIP-14 score } \\
\text { Mean(SD) }\end{array}$} & \multicolumn{3}{|c|}{ Difference (after-before) } \\
\hline & & & Mean(SD) & Effect size $^{a}$ & $p$-value \\
\hline $\mathrm{COHIP}-14$ & $37.5(7.9)$ & $45.2(7.7)$ & $7.7(8.1)$ & 1.0 & $<0.001^{*}$ \\
\hline Oral Health subscale $(\mathrm{OH})$ & $26.0(5.4)$ & $31.9(5.7)$ & $5.8(6.0)$ & 1.0 & $<0.001^{*}$ \\
\hline Pain/tooth ache & $2.7(1.0)$ & $3.4(0.8)$ & $0.7(1.2)$ & 0.6 & \\
\hline Breathing through mouth & $2.0(1.1)$ & $2.5(1.2)$ & $0.5(1.2)$ & 0.4 & \\
\hline Discoloration of teeth & $1.9(1.4)$ & $3.4(1.1)$ & $1.5(1.6)$ & 0.9 & \\
\hline Crooked teeth or spaces & $2.5(1.5)$ & $3.5(1.0)$ & $1.0(1.6)$ & 0.6 & \\
\hline Sores or sore spots & $3.4(0.8)$ & $3.6(0.7)$ & $0.2(0.8)$ & 0.3 & \\
\hline Bad breath & $2.1(1.3)$ & $2.8(1.2)$ & $0.6(1.2)$ & 0.5 & \\
\hline Bleeding gums & $3.2(1.0)$ & $3.3(0.9)$ & $0.1(0.9)$ & 0.1 & \\
\hline Food sticking & $2.1(1.0)$ & $2.5(1.1)$ & $0.4(1.3)$ & 0.3 & \\
\hline Sensitivity to hot/cold & $3.1(1.0)$ & $3.6(0.7)$ & $0.5(1.2)$ & 0.4 & \\
\hline Dry mouth & $3.1(1.1)$ & $3.3(0.9)$ & $0.2(1.0)$ & 0.2 & \\
\hline Functional Limitations subscale (FL) & $11.4(4.0)$ & 13.3(3.0) & $1.9(3.6)$ & 0.5 & $<0.001^{*}$ \\
\hline Trouble chewing firm foods & $2.3(1.5)$ & 2.9(1.4) & $0.6(1.5)$ & 0.4 & \\
\hline Difficulty eating & 2.8(1.3) & $3.3(1.0)$ & $0.6(1.3)$ & 0.5 & \\
\hline Trouble sleeping due to teeth/face & 3.6(0.8) & $3.9(0.4)$ & $0.3(0.8)$ & 0.4 & \\
\hline Difficulty keeping teeth clean & $2.8(1.4)$ & $3.2(1.2)$ & $0.4(1.4)$ & 0.3 & \\
\hline
\end{tabular}


Table 2 Prevalence and mean values of the 12-item Family impact scale (FIS-12) scores before and after dental treatment (average $2.4 \pm 1.7$ months' follow-up period) $(n=93)$

\begin{tabular}{|c|c|c|c|c|c|}
\hline & \multirow{2}{*}{$\begin{array}{l}\text { Before FIS-12 score } \\
\text { Mean(SD) }\end{array}$} & \multirow{2}{*}{$\begin{array}{l}\text { After FIS-12 score } \\
\text { Mean(SD) }\end{array}$} & \multicolumn{3}{|c|}{ Difference (after-before) } \\
\hline & & & Mean(SD) & Effect size $^{a}$ & $\overline{p \text {-value }}$ \\
\hline FIS-12 & $15.7(9.2)$ & $10.3(8.3)$ & $5.4(8.3)$ & 0.7 & $<0.001^{*}$ \\
\hline Parental/family Activity subscale (PA) & 7.4(4.8) & $4.7(4.5)$ & $2.7(4.7)$ & 0.6 & $<0.001^{*}$ \\
\hline Taken time off work & $0.6(1.0)$ & $0.3(0.7)$ & $0.3(1.0)$ & 0.3 & \\
\hline Required more attention & $2.8(1.3)$ & $1.9(1.5)$ & $0.8(1.5)$ & 0.5 & \\
\hline Had less time for yourself & $1.5(1.4)$ & $1.0(1.4)$ & $0.5(1.6)$ & 0.3 & \\
\hline Sleep disrupted & $1.5(1.3)$ & $0.8(1.1)$ & $0.7(1.2)$ & 0.6 & \\
\hline Family activity interrupted & $1.0(1.3)$ & $0.6(1.0)$ & $0.4(1.3)$ & 0.3 & \\
\hline Parental Emotion subscale (PE) & $6.1(3.8)$ & $4.0(3.4)$ & $2.1(3.2)$ & 0.7 & $<0.001^{*}$ \\
\hline Been upset & $1.3(1.2)$ & $0.9(1.0)$ & $0.4(1.1)$ & 0.4 & \\
\hline Felt guilty & $1.8(1.3)$ & $1.2(1.1)$ & $0.6(1.1)$ & 0.5 & \\
\hline Worried about less opportunity & $2.0(1.2)$ & $1.3(1.2)$ & $0.7(1.1)$ & 0.6 & \\
\hline Felt uncomfortable & $1.0(1.3)$ & $0.7(1.0)$ & $0.3(1.3)$ & 0.2 & \\
\hline Family Conflict subscale (FC) & $1.4(1.7)$ & $1.0(1.2)$ & $0.4(1.3)$ & 0.3 & $0.004^{*}$ \\
\hline Argued with child & $0.8(1.1)$ & $0.7(1.0)$ & $0.1(1.0)$ & 0.1 & \\
\hline Caused conflict in the family & $0.6(0.9)$ & $0.3(0.6)$ & $0.3(0.8)$ & 0.4 & \\
\hline Financial Burden subscale (FB) & $0.8(1.1)$ & $0.6(0.8)$ & $0.2(1.0)$ & 0.2 & 0.095 \\
\hline Cause financial difficulties & $0.8(1.1)$ & $0.6(0.8)$ & $0.2(1.0)$ & 0.2 & \\
\hline
\end{tabular}

Wilcoxon's signed-rank test

*Significant at $a=0.05$ level

${ }^{a}$ Calculated using Cohen's d (= difference / SD)

on a 6-point Likert scale from "Very bad" to "Very good".

\section{Statistical analysis}

Statistical analysis of the survey responses was performed using SPSS 21.0 (SPSS Inc., Chicago, IL, USA). The missing data was $4.39 \%$ of the total response. Before statistical analysis, the missing values of COHIP and FIS items were replaced by the variables' means to obtain sum scores. Since there were no statistically significant differences in the number of decayed teeth and the results of the OHRQoL questionnaire between general anesthesia and intravenous deep sedation, we have performed statistical analysis with the combined results. First, the Cronbach's alpha coefficient was used to measure internal consistency. As the Kolmogorov-Smirnov test indicated the COHIP-14 and FIS-12 scores did not follow a normal distribution, the Wilcoxon's signed-rank test was utilized to compare OHRQoL pre- and post-treatment. Cohen's d indicated the effect size and was calculated by dividing the average difference in OHRQoL scores between pre- and post-treatment by the standard deviation. An effect size of $0.2<\mathrm{d} \leq 0.5$ was considered small, $0.5<\mathrm{d} \leq 0.8$ was considered intermediate, and $d>0.8$ was considered large. To assess convergent validity, the partial Spearman correlation was examined between the COHIP and global ratings and between FIS score and global ratings. The Wilcoxon's rank-sum test was used to compare findings in patients with and without systemic disease and to compare individuals of different ages and genders. This test was also used to investigate effects of treatment variables, including number of decayed teeth, number of treated teeth and pulp treatment.

Finally, to understand the correlation between the utilized scales, a structural equation model was designed using IBM SPSS Amos 23.0.0 to build a Multi-indicator model. The hypotheses for the structural equation model were as follows. First, the subscales of COHIP and FIS could have different explanatory power on COHIP and FIS, and COHIP would have a significant explanatory power on FIS. The rationale for these hypotheses is that a chronic illness such as dental caries in children can affect the quality of life of the family, which is based on the family member's recognition of chronic diseases in children [13]. Second, the magnitude of the explanatory power in the structure equation model would be different depending on the presence or absence of systemic disease. Accordingly, the individual SEMs for patients with and without systemic disease were constructed by confirmative factor analysis. This study included COHIP and FIS subscales as observed variables and COHIP-14 and FIS-12 per se as latent variables. To assess the fitness of the structural equation model, the chi-square 
$p$-value, Goodness of Fit Index (GFI), and Normed Fit Index (NFI) were calculated. In general, if the GFI and NFI values are above 0.9 , the suggested model is appropriate and seems to have good explanatory power.

\section{Results}

Analysis was carried out on data from 93 pediatric patients (46 males and 47 females) and their primary caregivers. Among caregiver participants, 91 (97.8\%) were parents (81 mothers and ten fathers) and two (2.2\%) were grandmothers. A mean age of the 93 pediatric patients was $5.0 \pm 3.4$ years. There were 43 patients without systemic diseases (21 male and 22 female) with a mean age of $4.0 \pm 2.1$ years, while the remaining 50 patients had systemic diseases (25 male and 25 female), and a mean age of $5.9 \pm 3.9$ years. Patients with systemic disease were significantly older than those without $(p=0.012)$.

The average $\mathrm{dmft}$ index and the average number of treated teeth due to dental caries were $10.8 \pm 4.8$ and 8.8 \pm 4.4 , respectively. There was no significant difference in $\mathrm{dmft}$ index or number of treated teeth between the groups with $(10.6 \pm 4.5,8.7 \pm 4.7)$ and without $(11.0 \pm$ $5.1,9.0 \pm 4.0)$ systemic diseases $(p=0.648,0.640)$. Dental treatment included direct resin restoration, pulp treatment, prefabricated crown restoration and early extraction of carious teeth. The average number of teeth according to type of dental treatment was as follows: 5.6 \pm 3.3 for direct resin restoration, $2.6 \pm 2.8$ for pulp treatment, $2.7 \pm 2.9$ for prefabricated crown restoration, and $0.5 \pm 1.3$ for early extraction. There was no statistically significant difference between the two groups according to type of treatment.

In the 16 patients excluded from the analysis, the mean age, the average dmft index and the average number of treated teeth were $5.8 \pm 3.5,11.8 \pm 5.8$ and $8.8 \pm$ 4.4 , respectively. These results were not statistically different from the results of the 93 patients included in the analysis ( $p=0.426,0.409$ and 0.943 , respectively).

Cronbach's alpha coefficient, indicating internal consistency, for COHIP-14, OH, and FL were 0.737, 0.624 , and 0.769 , respectively. For FIS-12, PA, PE, and $\mathrm{FC}$, the values were $0.866,0.810,0.770$, and 0.532 , respectively.
COHIP scores were higher and FIS scores were lower post-treatment than pre-treatment. Therefore, the absolute value of the difference between pre- and post-treatment scores was used. Each of the item, preand post-treatment scores, as well as the difference in scores for COHIP-14 and FIS-12 are outlined in Tables 1 and 2. COHIP-14 and its subscale OH and FL scores were significantly and clinically improved at post-treatment (all $p<0.001$ and effect size $=1.0,1.0$, 0.5 respectively). Before dental treatment, the most frequent dental problem pointed out in $\mathrm{OH}$ was discoloration of the teeth $(37.6 \%)$, while discomfort during mastication $(33.3 \%)$ was indicated for FL.

FIS-12 and its subscale PA and PE scores were all significantly and clinically improved post-treatment (all $p<0.001$ and effect size $=0.7,0.6,0.7$ ). Before dental treatment, the most frequently reported concern in PA was "required more attention" (66.7\%), while that in PE was "worried about less opportunity in future due to dental problems" (37.6\%). In all the subcategories of $\mathrm{FC}$ and $\mathrm{FB}$, more than half of the responders reported "never" (57.0, 60.2, 55.9\%), or "almost never" (11.8, 20.4, 20.4\%).

As shown in Table 3, partial Spearman correlations indicated statistically significant associations between the COHIP-14/FIS-12 scores and the global oral health status and overall QoL both before and after dental treatment. For COHIP-14 before treatment, $\mathrm{r}(\mathrm{s})=0.438, p<0.001$ and $\mathrm{r}(\mathrm{s})=0.241, p=0.02$, respectively, and for FIS-12 before treatment, $\mathrm{r}(\mathrm{s})=-0.251, p=0.015, \mathrm{r}(\mathrm{s})=-0.391, \mathrm{p}<0.001$, respectively. For COHIP-14 after treatment, $\mathrm{r}(\mathrm{s})=0.429, p$ $<0.001$ and $\mathrm{r}(\mathrm{s})=0.287, p=0.005$, respectively, and for FIS-12 after treatment, $\mathrm{r}(\mathrm{s})=-0.396, \mathrm{p}<0.001, \mathrm{r}(\mathrm{s})=-$ $0.372, \mathrm{p}<0.001$, respectively. Correlations with the global oral health status were of moderate magnitude, and correlations with the overall QoL were of low magnitude.

As shown in Table 4, the presence of systemic disease accompanied lower OHRQoL. Gender did not play a significant role in pre- and post-treatment scores of either the COHIP-14 or FIS-12 or in improvement level $(p>$ 0.05). Age was not a significant factor for the FIS-12 score, but improvement in the COHIP-14 was significantly greater in patients aged 1-6 years than in those 7 years or older $(8.8 \pm 7.9,3.6 \pm 7.4, p=0.012)$. And COHIP-14 and FIS-12 were not associated with number

Table 3 Partial Spearman correlations between COHIP-14 and FIS-12 scores and global oral health status and overall quality of life

\begin{tabular}{|c|c|c|c|c|}
\hline & \multicolumn{2}{|c|}{ Global oral health status } & \multicolumn{2}{|c|}{ Overall quality of life } \\
\hline & $r(s)$ & $p$-value & $r(s)$ & $p$-value \\
\hline COHIP-14 before dental treatment & 0.438 & $<0.001$ & 0.241 & 0.02 \\
\hline FIS-12 before dental treatment & -0.251 & 0.015 & -0.391 & $<0.001$ \\
\hline COHIP-14 after dental treatment & 0.429 & $<0.001$ & 0.287 & 0.005 \\
\hline FIS-12 after dental treatment & -0.396 & $<0.001$ & -0.372 & $<0.001$ \\
\hline
\end{tabular}


Table $4 \mathrm{COHIP}$ and FIS scores according to gender, age, and medical condition of patients

\begin{tabular}{|c|c|c|c|c|c|c|c|}
\hline & & \multicolumn{2}{|l|}{ Gender } & \multicolumn{2}{|l|}{ Age } & \multicolumn{2}{|c|}{ Systemic Disease } \\
\hline & & Male & $\overline{\text { Female }}$ & $<7$ years & $\geq 7$ years & Healthy $^{a}$ & Diseased $^{k}$ \\
\hline & & \multicolumn{6}{|l|}{ Mean(SD) } \\
\hline \multirow[t]{3}{*}{$\mathrm{COHIP}-14$} & $B$ & $38.0(7.7)$ & $37.0(7.1)$ & $36.9(7.8)$ & 39.6(8.3) & $40.2(6.1)$ & $34.9(8.6)^{*}$ \\
\hline & A & $45.6(7.2)$ & $44.7(8.3)$ & $45.7(7.4)$ & $43.3(9.0)$ & $47.2(6.7)$ & $43.3(8.2)^{*}$ \\
\hline & $\mathrm{D}$ & $7.7(7.6)$ & $7.7(8.6)$ & $8.8(7.9)$ & $3.6(7.4)^{*}$ & $7.0(6.9)$ & $8.4(9.1)$ \\
\hline \multirow[t]{3}{*}{ FIS-12 } & B & 16.0(9.3) & $15.4(9.2)$ & 15.2(9.4) & $17.5(8.2)$ & 11.5(6.9) & $19.4(9.4)^{*}$ \\
\hline & A & 10.2(8.6) & 10.4(8.1) & $9.4(8.0)$ & 13.5(8.9) & $6.5(5.5)$ & $13.7(8.9)^{*}$ \\
\hline & D & $5.7(6.8)$ & $5.0(9.5)$ & $5.8(8.1)$ & $4.0(8.8)$ & $5.0(5.0)$ & $5.7(10.3)$ \\
\hline
\end{tabular}

Wilcoxon's rank-sum test

B: Before treatment

A: After treatment

$D$ in COHIP-14: Difference between $B$ and $A(A-B)$

$D$ in FIS-12: Difference between $B$ and $A(B-A)$

Healthy ${ }^{a}$ : Patients without systemic disease

Diseased ${ }^{\text {b }}$ Patients with systemic disease

*: Significantly different between groups $(p<0.05)$

of decayed teeth, number of treated teeth, and pulp treatment before and after dental treatment, respectively (all $p>0.05$ ). But more than five treated teeth and pulp treatment resulted in greater improvement in the COHIP-14 score ( $p=0.016$ and 0.024 , respectively).

Figures 1 and 2 shows the structure equation model flow-chart for pediatric patients without and with systemic disease respectively, as affected by COHIP and FIS variables. The coefficients estimated in this model represent the degree of explanatory power of the independent variable on the dependent variable, which indicates the degree to which the increment of one unit in independent variable changes the dependent variable including the error term. If the value is large, it has stronger explanatory power. The COHIP-14 score negatively affected the FIS-12 score, with explanatory power of 44.6 and $65.3 \%$ respectively. The reason for the negative direction is that higher COHIP and lower FIS scores indicate better OHRQoL. The magnitude of the explanatory power between COHIP and COHIP subscales and between FIS and FIS subscales was also greater in patients with systemic disease compared to patients without systemic disease. The chi-square test $p$-value, the GFI score and the NFI score were $0.807,0.972$, and 0.937 in the former model and were $0.060,0.917$, and 0.904 in the latter model, respectively. These results indicate exceptional fitness and explanatory power of the models.

\section{Discussion}

This is the first study to examine the potential association between dental treatment and OHRQoL in pediatric patients in Korea, using a Korean version of the COHIP, which is the only questionnaire that has undergone reliability and validity testing in Korean pediatric patients [4]. OHRQoL is a subjective concept that relies strongly on patient's awareness. Particularly in pediatric patients, teeth and facial development as well as psychological development vary markedly with age. The age of 6 years marks the beginning of abstract thinking and self-concept [23], and the understanding of even basic health concepts may be problematic in younger aged children, like the subjects of this study [24]. And pediatric patients with systemic disease often exhibit negative behavioral patterns during dental treatment due to the previous experiences in the medical hospital. They may also exhibit cognitive impairment,

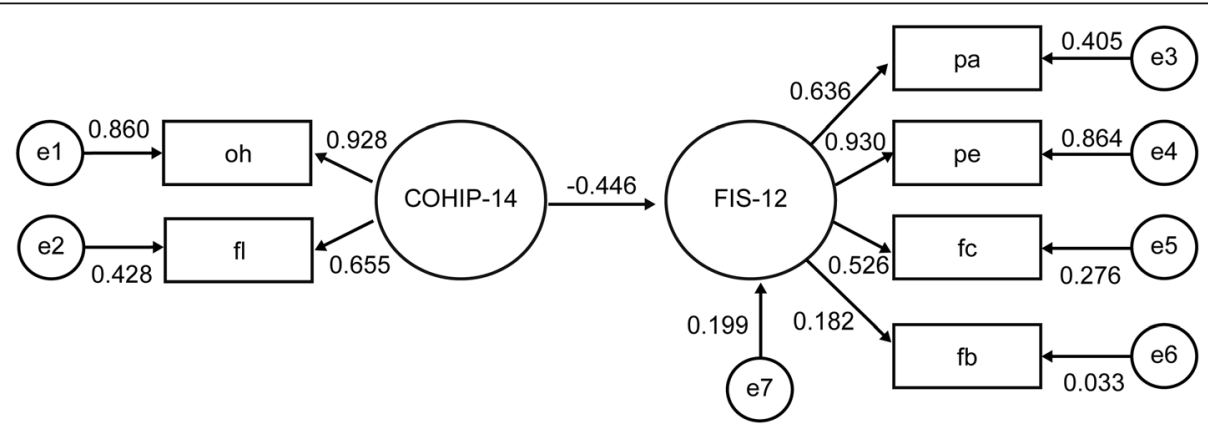

Fig. 1 Structure Equation Model of COHIP and FIS in pediatric patients without systemic disease 


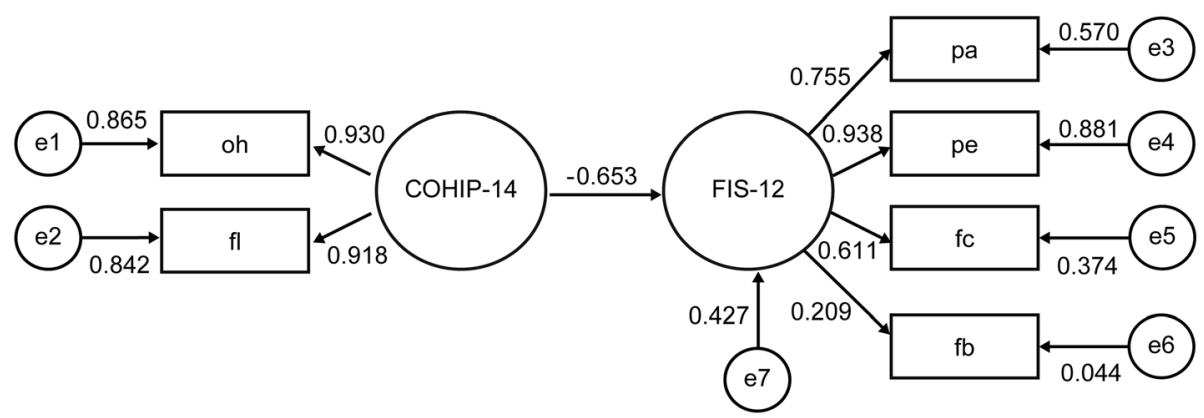

Fig. 2 Structure Equation Model of COHIP and FIS in pediatric patients with systemic disease

which makes it difficult to understand their cognitive processes and consequently results in unreliable measurement of QoL [7]. Therefore, studies on pediatric patients' OHRQoL often rely on the awareness of their primary caregivers [25]. Previous studies have shown that there was greater agreement for observable oral conditions and lesser agreement for non-observable oral conditions between ratings of children's OHRQoL made by parents and the children themselves [25]. In this context, the COHIP-14 and FIS-12 questionnaires used in this study were shortened from the original measurements for the primary caregiver to respond more appropriately. In order to supplement the modifications of the items, internal consistency and convergent validity were confirmed in this study.

The COHIP and FIS scores of pediatric patients were significantly and clinically improved after dental treatments under general anesthesia or intravenous deep sedation (Tables 1 and 2). These results were in agreement with those of previous studies that reported reduced OHRQoL due to a large number of dental caries $[5,6,26]$ and assessed the OHRQoL of pediatric patients treated under general anesthesia [7-9, 16, 27-29]. These previous studies showed statistical and clinical improvements in all subscales including oral symptoms and function. And the effect size of FL was lower than that of $\mathrm{OH}$, this is probably because the caregivers might have difficulties to recognize oral function objectively. This tendency is also observed in previous studies in which the caregivers responded to the questionnaire [7-9, 27, 29]. In contrast to these consistent results about dental caries on OHRQoL, the results of dental trauma and malocclusion, which are also common oral disorders in young pediatric patients, exhibited somewhat conflicting results [26, 30-32]. Overall, dental caries seems to have a greater impact on OHRQoL than dental trauma or malocclusion in young pediatric patients. This is likely because the OHRQoL questionnaires for young children are mostly completed by their caregivers, and dental caries in children are strongly influenced by the caregiver's daily oral hygiene care, but the trauma and malocclusion are not directly related to the caregiver's daily care.

Among the items in the COHIP, the most evident improvements were reported in the items of "discoloration of teeth" and "difficulty chewing firm food" before dental treatment, which are easy-to-notice changes for primary caregivers. This observation was slightly different from what was reported by Ahn et al. [4], where improvement in discoloration was reported at a low frequency, while improvements in food sticking in the teeth, crooked teeth, spaces between teeth, and difficulty in maintaining oral hygiene had high frequency. The age difference in the patient cohorts, as well as the targets of the investigation, patients versus primary caregivers, could account for the differences observed.

According to the study by Abanto et al. [5], dental caries exhibited a negative impact on the total FIS score and PA, PE and FB subscales. These are similar to our results except for the FB subscales. In our study, there was little change observed in the FB subscale, with the most frequent responses being "never" or "almost never" in items of the FB subscale. In cases where treatment is carried out under general anesthesia or intravenous deep sedation at Seoul National University Hospital, there is a possibility that the primary caregivers could either bear the financial burden for the treatment or received financial support from outside organizations. Among the items in the FIS, most evident improvements in this study were reported in the items of "required more attention" and "worried about less opportunity". These results are also similar to those of Abanto et al..

The results of Table 4 indicate that OHRQoL is lower in patients with systemic disease before and after dental treatment. These findings were in accordance with previous reports that found that patients with systemic diseases, such as cerebral palsy [17, 33], autism [34, 35], cancer [10, 36], and craniofacial anomalies [37], suffered from a lower OHRQoL.

Gender was not an important factor in OHRQoL, in agreement with reports by Broder et al. in 2007 and de Paula et al. in 2015 [22, 38]. Greater improvement in the 
COHIP score in patients aged 6 years or younger may be related to the significantly higher average number of treated teeth compared to patients aged 7 years or older (9.9 vs. $4.9, p<0.001)$. And this is consistent with the results that a large number of treated teeth and pulp treatments showed greater improvement in COHIP-14. A previous study reported that age is not an important factor, but that study did not consider the number of treated teeth when considering the effect of age [6].

The COHIP-14 appears to have a greater impact on the FIS-12 in patients with systemic disease (Figs. 1 and 2). Therefore, the diagnosis and understanding of oral health would exert a greater impact on the family's OHRQoL for patients with systemic disease. In other words, dental treatment and improvement in oral health can result in an overall increase in the OHRQoL of families of patients with systemic disease.

General anesthesia or intravenous deep sedation was performed by a single anesthesiologist. However, dental treatment was performed by five professors in pediatric dentistry working at Seoul National University Dental Hospital. Therefore, the follow-up period varied among the dentists, resulting in inconsistent time-lapses between pre- and post-treatment surveys. To minimize the effect of the inconsistencies, only cases in which the duration between surveys was less than 6 months were included for analysis. In addition, we removed several items from original COHIP and FIS questionnaires to compensate the differences of patient age and respondents to the questionnaire. Despite of the validity and reliability tests conducted in this study, the COHIP-14 and FIS-12 were not fully validated. And the magnitude of correlations between the COHIP-14/FIS-12 and the overall QoL were low. This is probably because more than half of the patients had systemic disease, and systemic disease itself, apart from oral health status, could have a negative impact on the overall QoL. Therefore, further research to confirm the overall reliability and validity of COHIP-14 and FIS-12 are required. A difference in the mean age of patients with and without systemic disease and the fact that there was no equivalent cohort of children with similar systemic condition who were treated without general anesthesia or intravenous deep sedation may be other confounders.

\section{Conclusion}

Dental treatment under either general anesthesia or intravenous deep sedation can improve the OHRQoL in Korean pediatric patients, which can be recognized by their primary caregivers. Systemic disease results in reduced OHRQoL, and the COHIP-14 appears to have a greater impact on the FIS-12 in patients with systemic disease than on patients without. In other words, the impact on the OHRQoL of the family is more pronounced in patients with systemic diseases, and thus treating dental caries in these patients will greatly improve the OHRQoL of the family members.

\begin{abstract}
Abbreviations
COHIP-14: Child oral health impact profile; FB: Financial burden subscale; FC: Family conflict subscale; FIS-12: Family impact Scale; FL: Functional limitation subscale; GFI: Goodness of fit index; NFI: Normed fit index; $\mathrm{OH}$ : Oral Health subscale; OHRQoL: Oral health-related quality of life; PA: Parental/family activity subscale; PE: Parental emotion subscale
\end{abstract}

\section{Funding}

This research was supported by a grant of the Korea Health Technology R\&D Project through the Korea Health Industry Development Institute (KHIDI), funded by the Ministry of Health \& Welfare, Republic of Korea (grant number: HI15(1503).

Availability of data and materials

The datasets used and/or analyzed during the current study are available from the corresponding author on reasonable request.

\section{Authors' contributions}

JS and YK conceived the ideas, TS is the anesthesiologist who performed general anesthesia and intravenous deep sedation in this study, and contributed to collect anesthesia related data. JS and $\mathrm{HH}$ contributed to statistical analysis of the collected data and led the writing. All authors have revised the manuscript, and have approved the final manuscript prior to its submission.

\section{Authors' information}

Dr. Ji-Soo Song is a specialist of pediatric dentistry and a clinical professor in the department of pediatric dentistry, Seoul National University Dental Hospital.

Dr. Hong-Keun Hyun is a specialist of pediatric dentistry and an associate professor in the department of pediatric Dentistry, dental research institute, school of dentistry, Seoul National University, Seoul National University Dental Hospital.

Dr. Teo Jeon Shin is an anesthesiologist and an associate professor in the department of pediatric Dentistry, dental research institute, school of dentistry, Seoul National University, Seoul National University Dental Hospital. Dr. Young-Jae Kim is a specialist of pediatric dentistry, a professor and the chairman in the department of pediatric Dentistry, dental research institute, school of dentistry, Seoul National University, Seoul National University Dental Hospital.

\section{Ethics approval and consent to participate}

The study was performed with the approval of the Seoul National University Dental Hospital Research Ethics Board (IRB Number: CRI12006), and we fully explained the study to the primary caregivers only if they were the legal guardians of the patients and only included participants with written consent on the day of treatment.

\section{Competing interests}

The authors declare that they have no competing interests.

\section{Publisher's Note}

Springer Nature remains neutral with regard to jurisdictional claims in published maps and institutional affiliations.

\section{Author details}

${ }^{1}$ Department of Pediatric Dentistry, Seoul National University Dental Hospital, 101, Daehakno, Jongno-gu, 03080 Seoul, Republic of Korea. ${ }^{2}$ Department of Pediatric Dentistry, Dental Research Institute, School of Dentistry, Seoul National University, Seoul National University Dental Hospital, 101, Daehakno, Jongno-gu, 03080 Seoul, Republic of Korea. 
Received: 29 September 2017 Accepted: 18 May 2018

Published online: 29 May 2018

\section{References}

1. Kassebaum NJ, Smith AGC, Bernabe E, et al. Global, regional, and national prevalence, incidence, and disability-adjusted life years for oral conditions for 195 countries, 1990-2015: a systematic analysis for the global burden of diseases, injuries, and risk factors. J Dent Res. 2017;96:380-7.

2. Ministry of Health and Welfare. The report of Korean National Oral Health Survey in 2012. Available in Korean at: "http://www.mohw.go.kr/react/jb/ sjb030301vw.jsp?PAR_MENU_ID=03\&MENU_ID=032901\&CONT_SEQ= 337111\&page=1". Accessed: 2018-05-23. (Archived by WebCite ${ }^{\oplus}$ at "http:// www.webcitation.org/6zcipMdG6").

3. Sischo L, Broder HL. Oral health-related quality of life: what, why, how, and future implications. J Dent Res. 2011:90:1264-70.

4. Ahn YS, Kim HY, Hong SM, Patton LL, Kim JH, Noh HJ. Validation of a Korean version of the child oral health impact profile (COHIP) among 8- to 15-year-old school children. Int J Paediatr Dent. 2012;22:292-301.

5. Abanto J, Paiva SM, Raggio DP, Celiberti P, Aldrigui JM, Bonecker M. The impact of dental caries and trauma in children on family quality of life. Community Dent Oral Epidemiol. 2012;40:323-31.

6. Acharya S, Tandon S. The effect of early childhood caries on the quality of life of children and their parents. Contemp Clin Dent. 2011;2:98-101.

7. Jabarifar SE, Eshghi AR, Shabanian M, Ahmad S. Changes in children's oral health related quality of life following dental treatment under general anesthesia. Dent Res J. 2009:6:13-6.

8. Gaynor WN, Thomson WM. Changes in young children's OHRQoL after dental treatment under general anaesthesia. Int J Paediatr Dent. 2012;22: 258-64.

9. Yawary R, Anthonappa RP, Ekambaram M, McGrath C, King NM. Changes in the oral health-related quality of life in children following comprehensive oral rehabilitation under general anaesthesia. Int J Paediatr Dent. 2016;26: 322-9.

10. Sheiham A. Dental caries affects body weight, growth and quality of life in pre-school children. Br Dent J. 2006;201:625-6.

11. BaniHani A, Deery C, Toumba J, Munyombwe T, Duggal M. The impact of dental caries and its treatment by conventional or biological approaches on the oral health-related quality of life of children and carers. Int J Paediatr Dent. 2018;28:266-76.

12. Amin MS, Harrison RL. Understanding parents' oral health behaviors for their young children. Qual Health Res. 2009;19:116-27.

13. Locker D, Jokovic A, Stephens M, Kenny D, Tompson B, Guyatt G. Family impact of child oral and oro-facial conditions. Community Dent Oral Epidemiol. 2002;30:438-48.

14. Pahel BT, Rozier RG, Slade GD. Parental perceptions of children's oral health: the early childhood oral health impact scale (ECOHIS). Health Qual Life Outcomes. 2007;5:6-10.

15. McMillan AS, Pow EH, Leung WK, Wong MC, Kwong DL. Oral health-related quality of life in southern Chinese following radiotherapy for nasopharyngeal carcinoma. J Oral Rehabil. 2004;31:600-8.

16. Baens-Ferrer C, Roseman MM, Dumas HM, Haley SM. Parental perceptions of oral health-related quality of life for children with special needs: impact of oral rehabilitation under general anesthesia. Pediatr Dent. 2005;27:137-42.

17. Abanto J, Ortega AO, Raggio DP, Bonecker M, Mendes FM, Ciamponi AL. Impact of oral diseases and disorders on oral-health-related quality of life of children with cerebral palsy. Spec Care Dent. 2014;34:56-63.

18. Espinoza KM, Heaton LJ. Communicating with patients with special health care needs. Dent Clin N Am. 2016;60:693-705.

19. American Academy of Pediatric Dentistry. Management of dental patients with special health care needs. Pediatr Dent. 2017;39:229-34.

20. Glassman P. Interventions focusing on children with special health care needs. Dent Clin N Am. 2017;61:565-76.

21. Chang J, Patton LL, Kim HY. Impact of dental treatment under general anesthesia on the oral health-related quality of life of adolescents and adults with special needs. Eur J Oral Sci. 2014;122:363-71.

22. Broder HL, Wilson-Genderson M. Reliability and convergent and discriminant validity of the child oral health impact profile (COHIP Child's version). Community Dent Oral Epidemiol. 2007;35(Suppl 1):20-31.

23. Jokovic A, Locker D, Stephens M, Kenny D, Tompson B, Guyatt G. Validity and reliability of a questionnaire for measuring child oral-health-related quality of life. J Dent Res. 2002;81:459-63.
24. Tsakos G, Blair YI, Yusuf H, Wright W, Watt RG, Macpherson LM. Developing a new self-reported scale of oral health outcomes for 5-year-old children (SOHO-5). Health Qual Life Outcomes. 2012;10:62.

25. Eiser C, Morse R. Can parents rate their child's health-related quality of life? Results of a systematic review. Quality Life Res. 2001;10:347-57.

26. Abanto J, Carvalho TS, Mendes FM, Wanderley MT, Bonecker M, Raggio DP. Impact of oral diseases and disorders on oral health-related quality of life of preschool children. Community Dent Oral Epidemiol. 2011;39:105-14.

27. Malden PE, Thomson WM, Jokovic A, Locker D. Changes in parent-assessed oral health-related quality of life among young children following dental treatment under general anaesthetic. Community Dent Oral Epidemiol. 2008;36:108-17.

28. Jankauskiene B, Narbutaite J. Changes in oral health-related quality of life among children following dental treatment under general anaesthesia. A systematic review. Stomatologija. 2010;12:60-4.

29. Ridell K, Borgstrom M, Lager E, Magnusson G, Brogardh-Roth S, Matsson L. Oral health-related quality-of-life in Swedish children before and after dental treatment under general anesthesia. Acta Odontol Scand. 2015;73:1-7.

30. Carvalho AC, Paiva SM, Viegas CM, Scarpelli AC, Ferreira FM, Pordeus IA. Impact of malocclusion on oral health-related quality of life among Brazilian preschool children: a population-based study. Braz Dent J. 2013;24:655-61.

31. Gomes MC, Pinto-Sarmento TC, Costa EM, Martins CC, Granville-Garcia AF, Paiva SM. Impact of oral health conditions on the quality of life of preschool children and their families: a cross-sectional study. Health Qual Life Outcomes. 2014;12:55.

32. Kramer PF, Feldens CA, Ferreira SH, Bervian J, Rodrigues PH, Peres MA Exploring the impact of oral diseases and disorders on quality of life of preschool children. Community Dent Oral Epidemiol. 2013;41:327-35.

33. Abanto J, Carvalho TS, Bonecker M, Ortega AO, Ciamponi AL, Raggio DP. Parental reports of the oral health-related quality of life of children with cerebral palsy. BMC Oral Health. 2012;12:15.

34. Pani SC, Mubaraki SA, Ahmed YT, Alturki RY, Almahfouz SF. Parental perceptions of the oral health-related quality of life of autistic children in Saudi Arabia. Spec Care Dent. 2013;33:8-12.

35. Yashoda R, Puranik MP. Oral health status and parental perception of child oral health related quality-of-life of children with autism in Bangalore, India. J Indian Soc Pedod Prev Dent. 2014;32:135-9.

36. Shavi GR, Thakur B, Bhambal A, Jain S, Singh V, Shukla A. Oral health related quality of life in patients of head and neck cancer attending cancer hospital of Bhopal City, India. J Int Oral Health. 2015;7:21-7.

37. Pope AW, Speltz ML. Research of psychosocial issues of children with craniofacial anomalies: progress and challenges. Cleft palate Craniofac J. 1997:34:371-3.

38. de Paula JS, Sarracini KL, Meneghim MC, et al. Longitudinal evaluation of the impact of dental caries treatment on oral health-related quality of life among schoolchildren. Eur J Oral Sci. 2015;123:173-8.

\section{Ready to submit your research? Choose BMC and benefit from:}

- fast, convenient online submission

- thorough peer review by experienced researchers in your field

- rapid publication on acceptance

- support for research data, including large and complex data types

- gold Open Access which fosters wider collaboration and increased citations

- maximum visibility for your research: over $100 \mathrm{M}$ website views per year

At BMC, research is always in progress.

Learn more biomedcentral.com/submissions 